\title{
Prevalence and antibiotic susceptibility of group A beta-haemolytic streptococcal isolates in children presenting with acute pharyngitis in Lusaka, Zambia
}

\author{
C Mwaba, ${ }^{1}$ MB ChB, MMed (Paed), Cert Paed Neph (SA), MPhil; R Nakazwe, ${ }^{2}$ BSc, MSc; \\ E Mpabalwani, ${ }^{1}$ BSc, MB ChB, MSc, MMed (Paed); C Lukwesa-Musyani, ${ }^{2}$ BSc, BSc HB, MB ChB, MPH, MSc; \\ J Mwansa, ${ }^{3}$ BSc HB, Dip Bact, PhD; J Chipeta, ${ }^{1}$ BSc HB, MB ChB, PhD \\ ${ }^{1}$ Department of Paediatrics and Child Health, School of Medicine, University of Zambia, Lusaka, Zambia \\ ${ }^{2}$ Department of Pathology, University Teaching Hospital, Lusaka, Zambia \\ ${ }^{3}$ Department of Microbiology, Lusaka Apex University, Lusaka, Zambia
}

Corresponding author: C Mwaba (chisambomwaba@yahoo.co.uk)

\begin{abstract}
Background. Group A beta-haemolytic streptococci (GABHS)-associated pharyngitis can complicate into rheumatic fever and rheumatic heart disease (RHD).

Objectives. To determine the prevalence and antibiotic susceptibility of GABHS isolates in children presenting with acute pharyngitis and assess the utility of Zambian Treatment Guideline (ZTG) criteria as a local clinical scoring system.

Methods. This descriptive cross-sectional study was conducted at the paediatric outpatient department of the University Teaching Hospital in Lusaka, Zambia. The study cohort, comprising children aged 3 - 15 years $(n=146)$, were recruited as presenting with symptoms of pharyngitis. The children underwent a clinical assessment that included a detailed case history, presenting symptoms and a throat swab that was subsequently cultured. Microbial isolates were typed and the antibiotic sensitivity of cultured GABHS to penicillin and erythromycin determined.

Results. GABHS were cultured from $22(15.1 \%)$ children within this study. All the GABHS isolates ( $n=22)$ were susceptible to penicillin G; however, $19 \%$ of isolates displayed reduced susceptibility to erythromycin. None of the ZTG criteria, when used individually, was sufficiently sensitive to detect GABHS pharyngitis among this cohort.

Conclusion. The prevalence of GABHS pharyngitis is similar that been described elsewhere. While GABHS remains highly susceptible to penicillin, which is used in the local RHD control programmes, concern remains for children treated with erythromycin owing to the resistance noted in some of the isolates. The ZTG clinical criteria displayed poor sensitivity in identifying GABHS pharyngitis. This has significant implications for effective diagnosis and treatment of pharyngitis and associated complications within this high RHD endemic area.
\end{abstract}

S Afr J Child Health 2020;14(2):99-103. https://doi.org/10.7196/SAJCH.2020.v14i2.1684

Group A beta-haemolytic streptococci (GABHS), or Streptococcus pyogenes, is the most important causative agent of bacterial pharyngitis because of the associated post infectious immunemediated complications of rheumatic fever and rheumatic heart disease (RHD). ${ }^{[1]}$ Children, aged 5 to 15 years, are most affected by GABHS pharyngitis, with the condition being rare in children younger than 3 years of age. ${ }^{[2]}$ This age group is also at most risk of developing rheumatic fever. ${ }^{[3]}$ There is an estimated prevalence of 15.3 million cases of RHD in the world with the highest prevalence reported in sub-Saharan Africa at 5.7 cases per thousand. ${ }^{[4]}$ RHD accounts for a large proportion (77\%) of morbidity due to cardiac diseases in children at the University Teaching Hospital (UTH). Adequate antibiotic treatment of the pharyngitis is known to prevent these complications. ${ }^{[1]}$

To the best of our knowledge, prior to this study there were no data on the prevalence of GABHS pharyngitis at UTH as throat cultures are not routinely performed. The Zambian Treatment Guidelines advocate the empirical treatment of GABHS pharyngitis based on the presence of four presenting clinical features, namely painful enlarged tonsils, tender enlarged cervical lymphadenopathy, absence of viral signs and presence of pharyngeal exudates, without providing a weighting for any of these factors. However, no clinical scoring system has ever been validated for use in the diagnosis of GABHS pharyngitis in Zambian children.

\section{Objectives}

To determine the prevalence of GABHS isolates in children presenting to UTH with pharyngitis, the antibiotic sensitivity of cultured pharyngeal GABHS isolates and the sensitivity of the Zambian Treatment Guideline for the empirical diagnosis of GABHS pharyngitis in children.

\section{Methods}

The study was conducted at the Paediatric Department, UTH, Lusaka, Zambia. Ethics approval was obtained prior to the start of the study from the institutional review committee of the School of Medicine, University of Zambia (ref. no. 004-02-11]/ IRB00001131). A study sample size of 140 was calculated, using Epi Info version 3.3.2 (Centers for Disease Control and Prevention, USA) at a confidence interval of $90 \%$, with a $p$-value of 0.05 and an estimated prevalence of $15 \%$ at a power of $80 \%$ and allowing a $5 \%$ loss to follow-up. ${ }^{[5]}$

The study included all children, aged 3 - 15 years, presenting to the outpatient department (OPD) with upper respiratory symptoms between April 2011 and September 2011. During this period a 
total of 12480 were seen in the OPD. A child was considered to have pharyngitis if they complained of upper respiratory symptoms and was found to have unequivocal erythema of the pharynx. Study exclusion criteria included children who had received antibiotics in the preceding 7 days and those known to have a history of rheumatic fever. Written informed consent to participate in the study was obtained from the children's parents, legal guardian or, in children older than 8 years, additional assent was sought where appropriate, from the children themselves. All those meeting the eligibility criteria were approached to join the study $(n=170)$; however, only 146 children were recruited to the study.

The clinical investigator obtained a standard medical history, including demographic data and presenting signs and symptoms, before performing a standard medical examination. The examination included a throat swab by standard clinical methods. The throat swab was placed in Amie's transport media tubes and transferred to the laboratory for microbiological analysis within 24 hours. The case history and examination results of each child were recorded on a standardised clinical case report form. Each child was assigned a unique study number which was inscribed on each of the data sheets as well as the laboratory request form.

The throat swabs were inoculated onto sheep's blood as well as chocolate agar and incubated at $37^{\circ} \mathrm{C}$ in $5 \% \mathrm{CO}_{2}$. The plates were examined for microbial growth after 24 and 48 hrs. Cultured microbial colonies were characterised based on colony morphology and the presence or absence of haemolytic zones of clearing on blood agar. All microbial isolates were assigned a unique laboratory serial number and preserved as cryostocks for future further analysis and typing. Beta-haemolytic isolates that were also identified as chains of Gram-positive cocci and catalase negative were purified and subjected to the presumptive test for GABHS. Briefly, a 0.4 unit bacitracin disc was placed on the blood agar plate inoculated with the purified isolate and incubated at $37^{\circ} \mathrm{C}$ in $5 \% \mathrm{CO}_{2}$ as described above. Group A streptococci are reported as those that were sensitive to bacitracin, while non-group A beta-haemolytic streptococci are resistant to bacitracin. ${ }^{[6]}$ The Streptex Rapid Latex Agglutination Test (Thermo Fisher Scientific, USA) was used for the serological identification of Lancefield groups A, B, C, D, F and G streptococci from the confirmed beta-haemolytic streptococci (BHS), including the bacitracin-resistant strains.

In the Zambian Treatment Guideline, the recommended drug for treatment of GABHS-associated pharyngitis is penicillin while for patients with penicillin allergy, erythromycin is advised. Antimicrobial susceptibility to penicillin and erythromycin, respectively, was performed on all the confirmed streptococcal isolates using the Kirby-Bauer disc diffusion method. ${ }^{[7]}$

The study-dependant variables were throat culture result and GABHS antibiotic susceptibility pattern. Independent variables included age, number of people in the household, number of shared rooms, presence of fever (defined as $>38.0^{\circ} \mathrm{C}$ ), presence of exudate (yellow/white matter seen on the pharynx or tonsils), presence of a tender node, including tenderness of an anterior cervical lymph node (LN) on palpation, presence of large node (an anterior cervical lymph node $1.5 \mathrm{~cm}$ in diameter), pain on swallowing (obtained from the patient history) and presence of enlarged tonsils.

A secondary data base was created on EpiData version 2.0 (EpiData Association, Denmark) and analysed using Epi Info version 3.3.2 and OpenEpi version 2.3 (openepi.com). ${ }^{[5]}$ Simple proportions were used to calculate the prevalence of GABHS pharyngitis in the study population as well as the antibiotic susceptibility of GABHS isolates. OpenEpi was used to calculate the antibiotic sensitivity and specificity as well as positive and negative predictive values of the various clinical predictive rules.

\section{Result}

The clinical characteristics of the study cohort included a mean (SD) age of $7.4(3.38)$ years and $80(56 \%)$ of the participants were girls. Most of the participants came from households with between five and 10 people, and the majority lived in homes that had three to five rooms (Table 1). The majority of the children were reported as having experienced fever at some point during their illness; however, only $18(12.6 \%)$ were actually found to have a temperature greater than $38^{\circ} \mathrm{C}$ on examination. Other symptoms experienced by the children included headache, abdominal pain and sore throat. Signs noted included rhinitis, conjunctivitis, scarlatiniform rash, exudates and tender cervical lymphadenopathy (Table 1). Two of the children who presentwed with a scarlatiniform rash and pharyngitis were subsequently diagnosed with rubella on analysis of serum immunoglobulin $\mathrm{M}$ titres.

In total, 76 microorganisms were cultured from the children in this study (Table 2), including 10 patient samples that cultured more than one microorganism. The majority of the isolates $(n=38$; $54 \%$ ) were BHS. However, GABHS was cultured from only 22 of the participants' throat swabs, thus accounting for a GABHS pharyngitis prevalence of $15.1 \%$. Of note is the fact that no group B streptococcus was isolated in our cohort of patients. Other putative pathogenic bacteria isolated included Staphylococcus aureus, Streptococcus pneumoniae, Neisseria spp. and Haemophilus spp.

Antimicrobial susceptibility testing showed that all the BHS isolates $(n=38)$ were susceptible to penicillin (Fig. 1). Of the 22 Lancefield group A isolates, 17 (81\%) were susceptible, three (14\%) were intermediate susceptible and one (5\%) was resistant to erythromycin. Among the Lancefield group C isolates $(n=12)$, nine $(75 \%)$ were susceptible, two (17\%) were intermediate susceptible and one (8\%) was resistant to erythromycin. All Lancefield group G $(n=4)$ and $\mathrm{D}(n=2)$ isolates were susceptible to erythromycin.

The present study found that the sensitivity and specificity of the various individual parameters, namely pharyngeal exudates (sensitivity $4.5 \%$; specificity $84 \%$ ), painful enlarged tonsils (sensitivity $40 \%$; specificity $59.7 \%$ ), fever (sensitivity $18 \%$; specificity $88.8 \%$ ), and tender cervical LNs (sensitivity $18 \%$; specificity $67.7 \%$ ) and absence of viral signs (sensitivity $36 \%$; specificity $58.8 \%$ ) varied substantially (Fig. 2). Furthermore, none of the children with GABHS pharyngitis had a combination of all five parameters occurring concurrently, making it impossible to assess the sensitivity of a combination of all five parameters occurring together.

\section{Discussion}

The prevalence of GABHS pharyngitis within this study was within the range reported in similar studies on the Indian subcontinent and in Alexandria (Egypt), Cape Town (South Africa) and Ethiopia. ${ }^{[8-12]}$ Low- to middle-income countries generally report GABHS pharyngitis prevalence rates of $14.9-20.7 \%$, high-income countries have prevalence rates of $22.6-26.1 \% \cdot{ }^{[4]}$ As poor socioeconomic conditions are associated with a higher prevalence of GABHS pharyngitis and rheumatic fever/ heart disease, this was attributed to skin-associated streptococcal infections playing a greater role in the aetiology of rheumatic fever in low-income communities than previously thought. ${ }^{[4,13]}$

In studies from Croatia, Cairo (Egypt) and Benin, however, the prevalence of GABHS pharyngitis was significantly higher with 
Table 1. Baseline characteristics of the participants and the association between GAS pharyngitis and exposure variables $(N=146)$

\begin{tabular}{|c|c|c|c|c|c|}
\hline Characteristic & Frequency, $n(\%)$ & Crude OR $(90 \%$ CI $)$ & $p$-value & aOR $(90 \% \mathrm{CI})$ & $p$-value \\
\hline \multicolumn{6}{|l|}{ Age (years) } \\
\hline$<5$ & $34(24)$ & 1.00 & $\mathrm{n} / \mathrm{a}$ & 1.00 & $\mathrm{n} / \mathrm{a}$ \\
\hline $5-10$ & $79(55)$ & $2.90(0.96-8.69)$ & 0.11 & $3.77(0.99-14.3)$ & 0.1 \\
\hline$>10$ & $30(21)$ & $0.86(0.21-3.49)$ & 0.86 & $1.4(0.2-7.4)$ & 0.73 \\
\hline \multicolumn{6}{|l|}{ Sex } \\
\hline Male & $64(44)$ & 1.00 & 0.00 & 1.00 & $\mathrm{n} / \mathrm{a}$ \\
\hline Female & $80(56)$ & $1.1(0.51-2.41)$ & 0.82 & $1.01(0.39-2.61)$ & 0.98 \\
\hline \multicolumn{6}{|l|}{ Household crowding (people) } \\
\hline$<5$ & $32(7)$ & 1.00 & 0.00 & 1.00 & $\mathrm{n} / \mathrm{a}$ \\
\hline $5-10$ & $102(87)$ & $1.85(0.62-5.46)$ & 0.40 & $1.35(0.40-4.54)$ & 0.68 \\
\hline \multicolumn{6}{|c|}{ Household ventilation (total number of rooms) } \\
\hline$<3$ & $26(7)$ & 1.00 & 0.00 & 1.00 & $\mathrm{n} / \mathrm{a}$ \\
\hline $3-5$ & $70(57)$ & $1.64(0.53-5.12)$ & 0.47 & $4.00(0.88-18.0)$ & 0.13 \\
\hline$>5$ & $45(36)$ & $1.05(0.30-3.60)$ & 0.95 & $2.00(0.43-9.7)$ & 0.44 \\
\hline \multicolumn{6}{|l|}{ Symptoms and signs } \\
\hline History of fever & $66(65)$ & $0.91(0.78-1.07)$ & 0.37 & $0.93(0.79-1.12)$ & 0.51 \\
\hline Headache & $56(42)$ & $1.18(0.47-2.93)$ & 0.72 & $0.63(0.06-5.98)$ & 0.69 \\
\hline Abdominal pain & $33(22.6)$ & $1.18(0.47-2.93)$ & 0.72 & $0.60(0.06-5.38)$ & 0.64 \\
\hline Sore throat & $83(56.8)$ & $1.12(0.51-2.40)$ & 0.81 & $0.79(0.28-1.81)$ & 0.55 \\
\hline $\begin{array}{l}\text { Presentation of fever } \\
\left(>38^{\circ} \mathrm{C}\right)\end{array}$ & $18(12.6)$ & $1.81(0.65-5.61)$ & 0.33 & $2.05(0.57-7.30)$ & 0.35 \\
\hline Cervical lymphadenopathy & $44(30.1)$ & $2.14(0.81-5.61)$ & 0.19 & $3.42(1.02-11.3)$ & 0.09 \\
\hline Rhinitis & $85(58)$ & $0.76(0.34-1.68)$ & 0.58 & $0.48(0.16-1.39)$ & 0.25 \\
\hline Conjunctivitis & $14(9.7)$ & $2.34(0.40-1.35)$ & 0.42 & $2.54(0.35-18.1)$ & 0.43 \\
\hline Scarletiniform rash & $15(10)$ & $2.67(0.46-15.3)$ & 0.35 & $2.69(0.40-18.0)$ & 0.39 \\
\hline Exudates & $20(13.7)$ & $3.80(0.67-2.14)$ & 0.20 & $3.0(0.45-20.6)$ & 0.33 \\
\hline Tender cervical LN & $14(28.9)$ & $0.11(0.11-1.21)$ & 0.07 & $0.10(0.01-1.25)$ & 0.07 \\
\hline
\end{tabular}

Table 2. Spectrum of the microorganisms isolated in this study $(N=76)$

\begin{tabular}{ll}
\hline Putative microbial identity & $\boldsymbol{n}(\%)^{*}$ \\
\hline Beta-haemolytic streptococci & $38(50)$ \\
Group A, $n$ & 22 \\
Group C, $n$ & 12 \\
Group G, $n$ & 4 \\
Haemophilus spp. & $15(20)$ \\
Alpha-haemolytic streptococci & $7(10)$ \\
Staphylococcus aureus & $5(7)$ \\
Neisseria spp. & $5(7)$ \\
Streptococcus pneumoniae & $4(5)$ \\
Group D (non-haemolytic) streptococci & $2(3)$ \\
*Unless otherwise specified. &
\end{tabular}

isolation rates of $42,42.2$ and $48.7 \%$, respectively. ${ }^{[14-16]}$ In contrast, a study from Mozambique reported a prevalence of $6.1 \%{ }^{[17]}$ It is known that geography, age, socio-economic conditions and season may influence the prevalence of GABHS pharyngitis infections, which may explain these disparities. ${ }^{[4,13]}$

BHS accounted for $50 \%$ of the cultured bacterial isolates in this study. While this is similar to that reported from Benin, ${ }^{[16]}$ other studies have reported lower proportions of BHS among cultured isolates. ${ }^{[10,18,19]}$ This illustrates the large proportion of bacterial pharyngitis in our cohort of children. In this study, similar to what has previously been reported, ${ }^{[10]}$ GABHS comprised the majority (64\%) of BHS isolates, while the most prevalent non-GABHS streptococcal isolates were groups C and G. This is in contrast to the much lower proportion (6.5\%) of non-group A streptococcal isolates reported in studies from the USA. ${ }^{[18,19]}$ Carriage studies in Africa have noted a predominance of these two streptococcal groups, suggesting that group $\mathrm{C}$ and $\mathrm{G}$ streptococci are far more important throat pathogens in the tropics. This has led to increased speculation, in contrast to what was previously thought, on whether group $\mathrm{G}$ and/or $\mathrm{C}$ streptococci may play a role in the pathogenesis of acute rheumatic fever. ${ }^{[20]}$

The only Haemophilus spp. isolated in this study were $H$. paraphrophilus and $H$. aphrophilus and these probably reflect throat carriage. The glaring absence of $H$. influenzae among the isolates may be explained by the fact that many of these children had received the conjugate $H$. influenzae type B (Hib) vaccine which reduces $H$. influenzae type $\mathrm{b}$ pharyngeal carriage rates. ${ }^{[21]}$

All the GAS isolates were susceptible to penicillin; however, up to $19 \%$ of the GAS isolates had either intermediate susceptibility or complete resistance to erythromycin. This phenomenon has also been reported for GAS isolates in Europe and the Far East, although the reported prevalence of erythromycin resistance was much higher. ${ }^{[22-26]}$ The Greek study reported that all the GABHS isolates that were resistant to erythromycin were also resistant to the antibiotics azithromycin and clarythromycin, and harboured macrolide resistance genes. ${ }^{[2]}$ The 


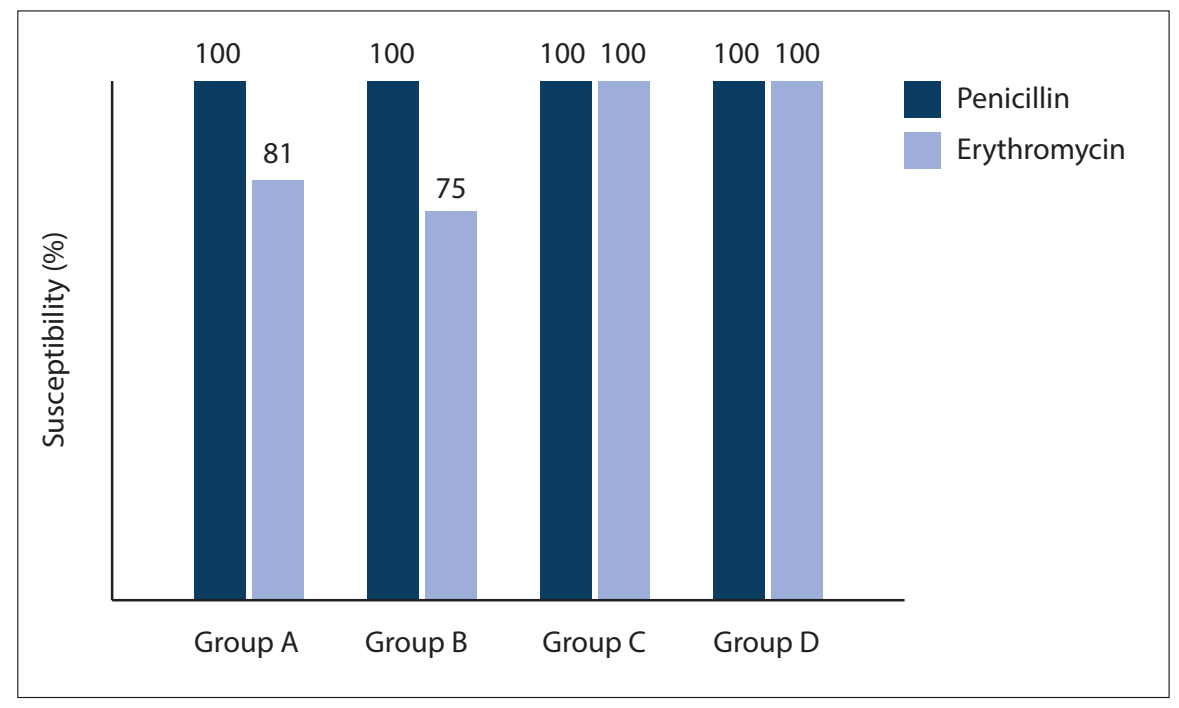

Fig. 1. Susceptibility of group A, C, D and G serogroups of streptococcal isolates to penicillin and erythromycin.

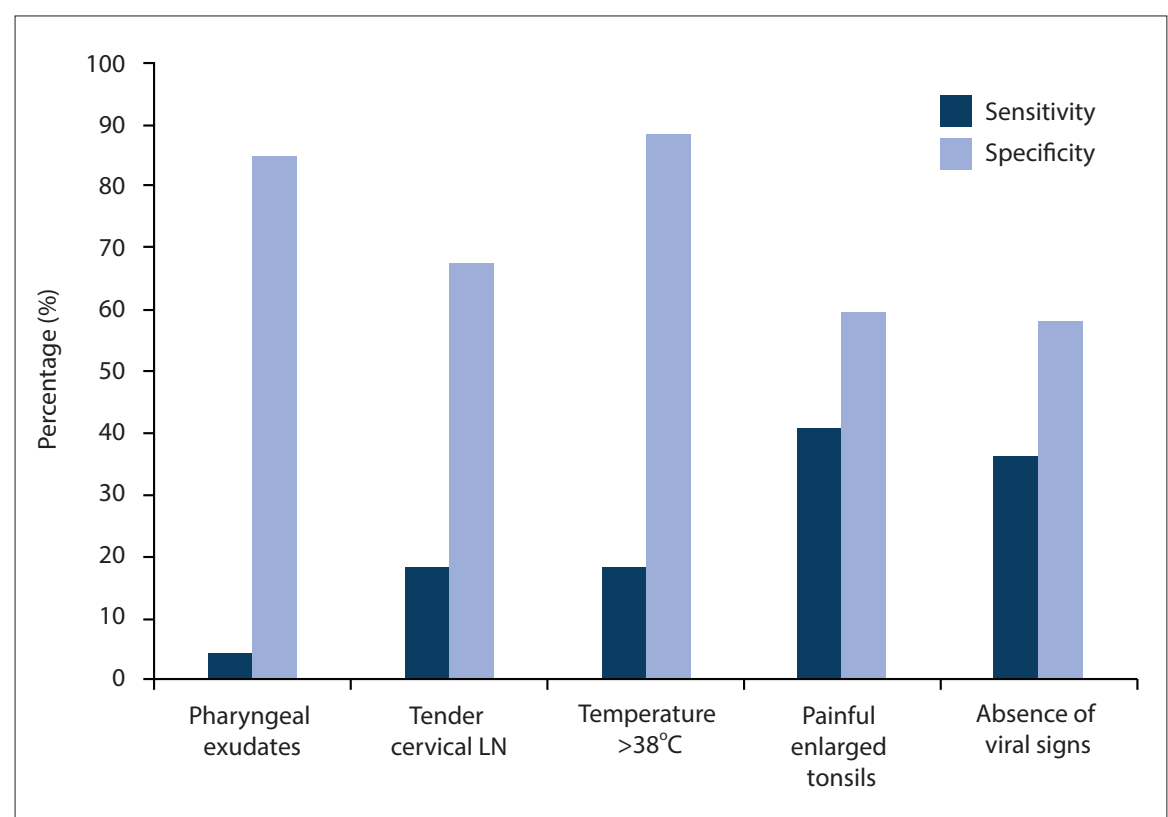

Fig. 2. The sensitivity and specificity of the five parameters on the Zambian Treatment Guidelines. (LN = lymph node.)

present study did not test the sensitivity of the cultured isolates to other macrolides nor was the mechanism of erythromycin resistance characterised. The implications of the observed resistance of GAS isolates to erythromycin are that children with GABHS pharyngitis and who are subsequently treated with erythromycin may still be at risk of developing rheumatic fever and consequently rheumatic heart disease. It would be prudent for the national RHD surveillance programme to keep tract of the evolution of macrolide resistance. All the Lancefield group D and $G$ isolates were susceptible to both penicillin and erythromycin, while only $75 \%$ of the group $\mathrm{C}$ isolates were susceptible to erythromycin. The country treatment guideline for the management of acute pharyngitis has a list of symptoms and signs that are considered suggestive of GABHS pharyngitis in a patient. However, the guideline does not suggest how many parameters should be present to make the diagnosis. When used individually, none of the five individual parameters, namely pharyngeal exudates, fever, tender cervical LN, absence of viral signs and painful enlarged tonsils, in the Zambian Treatment Guidelines criteria had sufficient sensitivity to predict a positive culture of GABHS. Thus no single criterion was sensitive enough to predict GABHS positivity on throat culture. None of the children with GABHS pharyngitis presented with a cluster of all the four parameters, therefore making it impossible to assess the sensitivity of a combination of the four parameters occurring concurrently. This could be due to our fairly small sample size as Steinhoff et al. ${ }^{[27]}$ had also reported a similarly low number of children displaying a combination of these signs and symptoms. This brings into question the prudence of relying solely on clinical features, whether individually or as part of a predictive rule, to make a diagnosis of streptococcal pharyngitis, especially in regions with high RHD prevalence. In a systematic review of clinical predictive rules, Shaikh et al. ${ }^{[28]}$ concluded that symptoms and signs, either individually or combined into prediction rules, cannot be used to definitively diagnose or rule out streptococcal pharyngitis.

\section{Study limitations and strengths}

This study has several limitations. As a hospital study and based on passive recruitment, there may have been selection bias in terms of the type of patients who presented to the hospital. Therefore, the data may have limited applicability to the general population. Secondly, this was a cross-sectional study so it is difficult to know for certain whether the GABHS isolates represent true GABHS pharyngitis as opposed to simple bacterial carriage in those who had coincidental viral pharyngitis. Furthermore, emm typing of the clinical isolates was not performed and it is hoped that future studies will include molecular characterisation as this will add insights into the predominant pharyngitis strains circulating within our population. This may help to identify prevalent GABHS strains that need to be the targeted for future vaccine development. Finally, our relatively small sample size may have affected our ability to accurately test the validity of the Zambian Treatment Guidelines.

The benefits of this study include a baseline clinical dataset to inform the appropriate antibiotic to use in the empirical treatment of suspected bacterial pharyngitis in this region. This study also provided evidence that the local clinical predictive tool has low sensitivity to diagnose children with GABHS pharyngitis and provides a basis upon which future studies can attempt to formulate locally validated clinical presumptive treatment guidelines.

\section{Conclusions}

The prevalence of GABHS in children presenting with pharyngitis to UTH, Zambia was $15.1 \%$. All cultured GABHS isolates, were sensitive to penicillin, although a proportion of isolates showed reduced susceptibility to erythromycin. This raises significant concerns about dissemination of drug-resistant strains 
and the effect upon treatment options in penicillin-sensitive children. The government-recommended clinical predictive guidelines were found not to be sufficiently sensitive for the diagnosis of GABHS pharyngitis. It is our opinion that perhaps approaches that focus on the development of cheaper and more sensitive point of care diagnostic methods such as rapid antigen tests for GABHS would form a better strategy for childhood pharyngitis management in our environment where up to $70 \%$ of all childhood cardiac morbidity is attributable to RHD.

This study had a high isolation rate of other non-group A streptococcal isolates which, like GABHS, also pose a risk of severe invasive disease. Therefore, in this clinical setting and given this high prevalence of bacterial pharyngitis, antibiotic use in most children presenting with pharyngitis remains a sound clinical decision.

Declaration. This work was submitted as part of a dissertation in partial fulfilment of the requirements for a MMed (Paed) at the University of Zambia Acknowledgements. Mr Francis Ngulube, Department of Pathology, University Teaching Hospital, Lusaka, for assistance with processing the specimens in the microbiology laboratory.

Author contributions. CM, EM and JC conceptualised the study. CM performed data collection and analysis and wrote the manuscript. RN carried out the laboratory tests. CL supervised the laboratory work. All authors contributed to the preparation of, and approved, the final version of the manuscript.

Funding. None.

Conflicts of interest. None.

1. Dajani A, Taubert K, Ferrieri P, Peter G, Shulman S. Treatment of acute streptococcal pharyngitis and prevention of rheumatic fever: A statement for health professionals. Committee on Rheumatic Fever, Endocarditis, and Kawasaki Disease of the Council on Cardiovascular Disease in the Young, the American Heart Association. Pediatrics 1995;96(4 Pt 1):758-764.

2. Oliver J, Malliya Wadu E, Pierse N, Moreland NJ, Williamson DA, Baker MG. Group A Streptococcus pharyngitis and pharyngeal carriage: A meta-analysis. PLoS Negl Trop Dis 2018;12(3): e0006335. https://doi.org/10.1371/journal. pntd.0006335

3. Carapetis JR, McDonald M, Wilson NJ. Acute rheumatic fever. Lancet 2005;366(9480):155-168. https://doi.org/10.1016/s0140-6736(05)66874-2

4. Ndiaye AG, Boye CS, Hounkponou E, Gueye FB, Badiane A. Antimicrobial susceptibility of select respiratory tract pathogens in Dakar, Senegal. J Infect Dev Ctries 2009;3(9):660-666. https://doi.org/10.3855/jidc.20

5. Sullivan KM, Dean A, Soe MM. OpenEpi: A web-based epidemiologic and statistical calculator for public health. Public Health Rep 2009;124(3):471-474. https://doi.org/10.1177/003335490912400320

6. Campos, J. Group A Streptococcus: Culture and non-culture tests. In: Garcia LS, Isenberg HD, eds. Clinical Microbiology Procedures Handbook, 3rd edition. Washington DC: American Society for Microbiology Press, 2010.

7. CLSI. Performance Standards for Antimicrobial Susceptibility Testing, 21st edition. CLSI document M100-S21. Wayne: Clinical and Laboratory Standards Institute, 2011

8. Nandi S, Kumar R, Ray P, Vohra H, Nirmal K, Ganguly NK. Group A streptococcal sore throat in a peri-urban population of northern India: A oneyear prospective study. Bull World Health Organ 2001;79(6):528-533.

9. Jain A, Shukla VK, Tiwari V, Kumar R. Antibiotic resistance pattern of group A beta-haemolytic streptococci isolated from north Indian children. Indian J Med Sci 2008;62(10):392-396.

10. Basilli A, Barakat S, Sawaf EL, Zaher S, Zakia, Din S. Identification of clinical criteria for group A beta haemolytic streptococcal pharyngitis in children living in a rheumatic fever endemic area. J Trop Pediatr 2002;48(5):285-293. https://doi.org/10.1093/tropej/48.5.285.
11. Engel ME, Cohen K, Gounden R, et al. The Cape Town decision rule for streptococcal pharyngitis in children. Pediatr Infect Dis J 2017;36(3):250-255. https://doi.org/10.1097/INF.0000000000001413

12. Tesfaw G, Kibru G, Mekonnen D, Abdissa A. Prevalence of group A betahaemolytic Streptococcus among children with pharyngitis in Jimma town, Southwest Ethiopia. EJENTAS 2015;16(1):35-40. https://doi.org/10.1016/j. ejenta.2015.02.001

13. Smeesters PR, Vergison A, Campos D, De Aguiar E, Deyi VYM, Van Meldern L. Differences between Belgian and Brazilian group A streptococcal epidemiologic landscape. PLoS ONE 2006;1(1):e10. https://doi.org/10.1371/ journal.pone.0000010

14. Rimoin A, Walker CF, Chitale RA, Hamza HS, Vince A, Gardovska D. Variation in clinical presentation of childhood group A streptococcal pharyngitis in four countries. J Trop Pediatr 2008 54(5):308-312. https://doi.org/10.1093/tropej/ fmm122

15. Abd El-Ghany SM, Abdelmaksoud AA, Saber SM, Abd El Hamid DH. Group A beta-hemolytic streptococcal pharyngitis and carriage rate among Egyptian children: A case-control study. Ann Saudi Med 2015;35(5):377-382. https://doi. org/10.5144/0256-4947.2015.377

16. Sadoh WE, Sadoh AE, Oladipo AO, Okunola OO. Bacterial isolates of tonsillitis and pharyngitis in a paediatric casualty setting. J Med Biomed Research 2008; 7(1-2):37-44. https://doi.org/10.4314/jmbr.v7i1-2.44555

17. Madeira G, Chicavel D, Munguambe A, Langa J, Mocumbi A. Streptococcal pharyngitis in children with painful throat: Missed opportunities for rheumatic heart disease prevention in endemic area of Africa. Cardiovasc Diagn Ther 2017;7(4):421-423. https://doi.org/10.21037/cdt.2017.05.07

18. Schwartz RH, Shulman ST. Group C and group G streptococci: In-office isolation from children and adolescents with pharyngitis. Clin Pediatr (Phila) 1986;25(10):496-502. https://doi.org/10.1177/000992288602501003

19. Zaoutis T, Attia M and Gross R, Klein J. The role of group C and group $\mathrm{G}$ streptococci in acute pharyngitis in children. Clin Microbiol Infect 2004;10(10):37-40. https://doi.org/10.1111/j.1469-0691.2004.00732.x

20. Hofkosh D, Wald ER, Chiponis DM. Prevalence of non-group-A betahemolytic streptococci in childhood pharyngitis. South Med J 1988;81(3):329331. https://doi.org/10.1097/00007611-198803000-00011

21. Mohle-Boetani JC, Aiello G, Breneman E, Deaver KA, Harvey C, Plikaytis BD. Carriage of Haemophilus influenzae type b in children after widespread vaccination with conjugate Haemophilus influenzae type b vaccines. Pediatr Infect Dis J 1993;12(7):589-593. https://doi.org/10.1097/00006454-19930700000009

22. Montagnani F, Stolzuoli L, Croci L, Rizzuti C. Erythromycin resistance in Streptococcus pyogenes and macrolide consumption in a central Italian region. Infection 2009;37(4):353-357. https://doi.org/10.1007/s15010-008-8023-1

23. Camponovo CR. In vitro susceptibility patterns of bacterial species isolated in outpatients of the Metropolitan Región, Chile, year 2007. Rev Chilena Infectol 2009;26(1):18-20. https://doi.org/S0716-10182009000100002

24. Gracia M, Diaz C, Coronel P, Gimeno M, García-Rodas R, Rodríguez-Cerrato V. Antimicrobial susceptibility of Streptococcus pyogenes in Central, Eastern, and Baltic European Countries, 2005 to 2006: The cefditoren surveillance program. Diagn Microbiol Infect Dis 2009;64(1):52-56. https://doi.org/10.1016/j. diagmicrobio.2008.12.018

25. Olivieri R, Morandi M, Zanachi A, Tordini G, Pozzi G, De Luca A. Evolution of macrolide resistance in Streptococcus pyogenes over 14 years in an area of central Italy. J Med Microbiol 2015;64(10):1186-1195. https://doi.org/ 10.1099/ jmm.0.000146.Epub2015 Jul 28

26. Syrogiannopoulos GA, Grivea IN, Fitoussi F, et al. High prevalence of erythromycin resistance of Streptococcus pyogenes in Greek children. Pediatr Infect Dis J 2001;20(9)863-868.

27. Steinhoff MC, Abd el Khalek KA, Khallaf N, Hamza HS, el Ayadi A, Orabi A. Effectiveness of clinical guidelines for the presumptive treatment of streptococcal pharyngitis in Egyptian children. Lancet 1997;350(9082):918921. htpps://doi.org/10.1016/SO140-6736(97)03317-5

28. Shaikh N, Swaminathan N, Hooper E. Accuracy and precision of the signs and symptoms of streptococcal pharyngitis in children: A systematic review. J Pediatr 2012;160(3):487-493.e3. https://doi.org/10.1016/j.jpeds.2011.09.011

Accepted 18 November 2019. 